University of Nebraska - Lincoln

DigitalCommons@University of Nebraska - Lincoln

6-16-2003

\title{
Nanoscale polarization manipulation and conductance switching in ultrathin films of a ferroelectric copolymer
}

Hongwei Qu

Florida International University, qu2@oakland.edu

Wei Yao

Florida International University, Miami, Florida

T. Garcia

Florida International University

Jiandi Zhang

Florida International University, jiandiz@lsu.edu

A.V. Sorokin

University of Nebraska-Lincoln

See next page for additional authors

Follow this and additional works at: https://digitalcommons.unl.edu/physicsdowben

Part of the Physics Commons

Qu, Hongwei; Yao, Wei; Garcia, T.; Zhang, Jiandi; Sorokin, A.V.; Ducharme, Stephen; Dowben, Peter A.; and Fridkin, V.M., "Nanoscale polarization manipulation and conductance switching in ultrathin films of a ferroelectric copolymer" (2003). Peter Dowben Publications. 11.

https://digitalcommons.unl.edu/physicsdowben/11

This Article is brought to you for free and open access by the Research Papers in Physics and Astronomy at DigitalCommons@University of Nebraska - Lincoln. It has been accepted for inclusion in Peter Dowben Publications by an authorized administrator of DigitalCommons@University of Nebraska - Lincoln. 


\section{Authors}

Hongwei Qu, Wei Yao, T. Garcia, Jiandi Zhang, A.V. Sorokin, Stephen Ducharme, Peter A. Dowben, and V.M. Fridkin 


\title{
Nanoscale polarization manipulation and conductance switching in ultrathin films of a ferroelectric copolymer
}

\author{
Hongwei Qu, Wei Yao, T. Garcia, and Jiandi Zhang ${ }^{\text {a) }}$ \\ Department of Physics, Florida International University, Miami, Florida 33199
}

A. V. Sorokin, S. Ducharme, and P. A. Dowben

Department of Physics and Astronomy, Behlen Laboratory of Physics, Center for Materials Research and Analysis, University of Nebraska-Lincoln, Lincoln, Nebraska 68588

\author{
V. M. Fridkin \\ Institute of Crystallography, The Russian Academy of Science, 117333, Moscow, Russia
}

(Received 25 February 2003; accepted 15 April 2003)

\begin{abstract}
We report the direct observation of induced molecular reorientation on a ferroelectric copolymer with a scanning tunneling microscope (STM). Ultrathin copolymer films of vinylidene fluoride $(70 \%)$ with trifluoroethylene $(30 \%)$ revealed a quasihexagonal close-packing structure with long-range polymer chain ordering. By flipping the polarity of the STM tip bias voltage, a reversal of local polarization was observed through an apparent lattice shift and was accompanied by an asymmetric "diode-like" character in tunneling current $I(V)$. These results clearly demonstrated conductance switching behavior on nanoscale with local polarization reversal. (C) 2003 American Institute of Physics. [DOI: 10.1063/1.1582366]
\end{abstract}

The manipulation of electronic properties on a singlemolecule scale has been a recent goal for molecular electronics. ${ }^{1}$ Conductance switching behaviors have been reported for molecular complexes. ${ }^{2-6}$ There is compelling evidence for reversible conductance transition associated with a molecular reorientation induced by a scanning tunneling microscope (STM). Molecular ferroelectrics, on the other hand, provide a framework for observing reversible conductance transitions. ${ }^{7}$ Ferroelectric polymers present an avenue for fabricating stable and ultrathin films, ${ }^{8-11}$ with sufficient crystallinity to visualize individual monomer behaviors. ${ }^{9,12} \mathrm{We}$ report here the STM-induced dipole reorientation on nanoscale in a ferroelectric copolymer material and the consequences on "local" perpendicular transport properties.

Recently, high-quality films of the ferroelectric copolymer of vinylidene fluoride with trifluoroethylene $[P(\mathrm{VDF}-\mathrm{TrFE})]$ have been fabricated using the LangmuirBlodgett (LB) monolayer-transfer technique, permitting precise control of nanoscale structure. ${ }^{8-10}$ The molecular units (or monomers) with net dipole moments pointing from the electronegative fluorine to the electropositive hydrogen form chains that crystallize into parallel rows. In both the bulk and thin films, a first-order ferroelectric-to-paraelectric phase transition occurs at $T_{\mathrm{C}} \sim 353 \mathrm{~K} \cdot{ }^{10,11}$ Associated with twodimensional ferroelectricity in thin LB crystallized films, ${ }^{8-13}$ there is no apparent critical thickness for ferroelectric behavior down to a film thickness of $1 \mathrm{~nm}$, much thinner than any previous ferroelectric films. ${ }^{14,15}$

High-quality thin films with two nominal monolayer (ML) thickness of $P(\mathrm{VDF}: 70 \%$-TrFE:30\%) for this study were grown on freshly cleaved, highly oriented pyrolytic graphite surfaces by horizontal LB deposition technique.,

\footnotetext{
a) Author to whom correspondence should be addressed; electronic mail: zhangj@fiu.edu
}

The samples were transferred into an UHV chamber with a base pressure of $2.0 \times 10^{-10}$ Torr for the measurements. The surface of the samples was cleaned by gentle annealing at the temperature of $\sim 420 \mathrm{~K}$ in UHV. The global ordering of the films was confirmed with low energy electron diffraction. ${ }^{18}$ All the STM measurements were performed at RT in an Omicron variable-temperature STM.

Figure 1 presents a typical atomically resolved STM image of a 2-ML $P(\mathrm{VDF}-\mathrm{TrFE})$ film at RT. Every protrusion represents a monomer either $-\mathrm{CH}_{2}-\mathrm{CF}_{2}-$ or $-\mathrm{CHF}-\mathrm{CF}_{2}-$ cluster, indistinguishable from the image. As shown in Fig. 1, these monomers arranged themselves in parallel rows/chains and a quasihexagonal close-packing structure, resembling a projection of the bulk structure in the ferroelectric phase. ${ }^{11,16}$ Through a careful calibration with a cleaved graphite surface imaged under exactly the same imaging conditions, we find that the intrachain monomer-monomer spacing $a$ on this 2-ML film is $2.4 \pm 0.1 \AA$, which followed the substrate lattice spacing and is consistent with the previous measured results. ${ }^{16}$ However, the average interchain spacing $b$ is only about $3.3 \pm 0.1 \AA$, much smaller than that in the bulk. In thick films or the bulk, the intra- and interchain spacing is 2.56 and $4.56 \AA$, respectively. ${ }^{17}$ This compression may be due to substrate induced in-plane strain.

We have directly employed the STM tip to induce a molecular reorientation and associated polarization change in the surface layer of the film by switching the polarity of tip bias $V_{\text {bias }}$. As shown in Fig. 2, the image was taken while flipping $V_{\text {bias }}$ at $\sim 1 / 3$ and at $\sim 2 / 3$ of the vertical sweep during scanning. An apparent step change in the image brightness caused by flipping bias has been removed from Fig. 2 . This is due to the asymmetric tunneling conductance with the bias polarity (see Fig. 3). The tunneling conductance appears higher with negative than equivalent positive tip bias. Some striking phenomena have been observed. (i) By flipping 


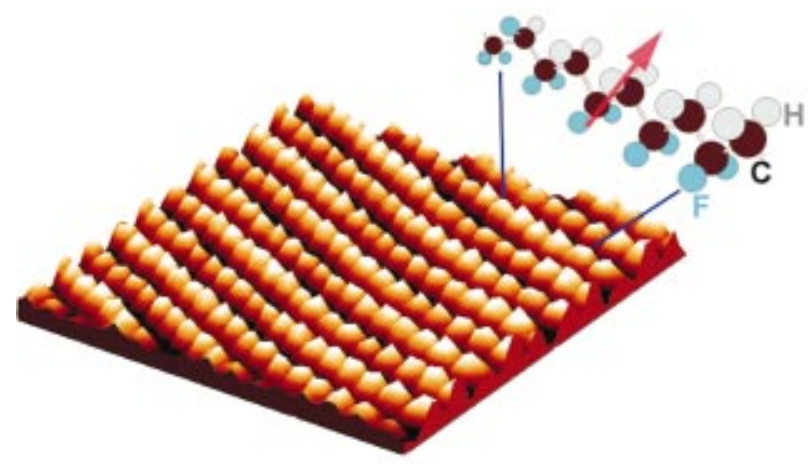

FIG. 1. (Color) A three-dimensional (3D) view of STM image on a 2-ML $P(\mathrm{VDF}-\mathrm{TrFE})$ copolymer film on graphite at RT. Every protrusion represents a $-\mathrm{CH}_{2}-\mathrm{CF}_{2}-$ or $-\mathrm{CHF}-\mathrm{CF}_{2}-$ cluster. The inset shows a ball model of the top-layer copolymer chain structure in ferroelectric phase. The image was taken with $V_{\text {bias }}=-0.36 \mathrm{~V}$ and $I=0.38 \mathrm{nA}$ (image size: $3.3 \mathrm{~nm}$ $\times 3.3 \mathrm{~nm})$.

$V_{\text {bias }}$, an apparent lattice shift by about a half of interchain spacing (i.e., $\sim 1.65 \AA$ ) occurred in the direction normal to copolymer chains (Fig. 2). Such a shift did not appear when the scanning direction is parallel to the copolymer chain axis. The shift is not an instrumental artifact, and cannot be reproduced on the nonpolar clean graphite surface. (ii) By keeping the ratio of $V_{\text {bias }} / I$ constant, such that the tip-sample gap remains roughly constant, it was found that the shift occurred only when $0.1 \mathrm{~V} \leqslant V_{\text {bias }} \leqslant 1.0 \mathrm{~V}$.

The shift could be a consequence of electronic state effects due to the difference between the occupied and unoccupied density of states without any rearrangement of local lattice structure or bonds. Any apparent shift in STM image by flipping bias should then closely depend on the bias voltage. However, the observed shift is nearly independent of the bias voltage in the flipping range of $0.1 \mathrm{~V} \leqslant V_{\text {bias }} \leqslant 1.0 \mathrm{~V}$. Thus, the observed shift is likely to be structural in origin.

The more plausible explanation for the shift is based upon a local dipole moment rotation as well as a change of local structure in the top layer of the film induced by the tip field, as schematically shown in Fig. 2. A negative tip bias

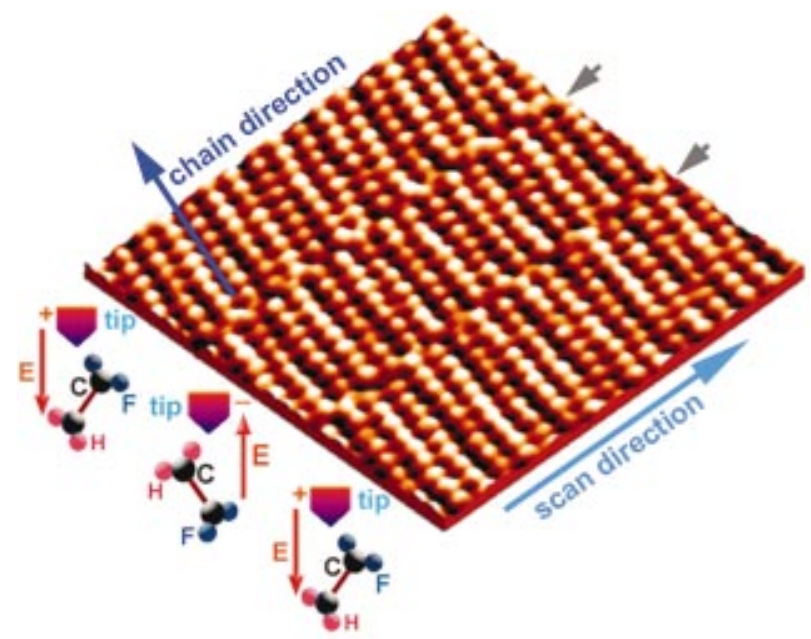

FIG. 2. (Color) A 3D view of STM image on a 2-ML P(VDF-TrFE) copolymer film at RT, while the polarity of $V_{\text {bias }}$ was flipped (as indicated by arrows) from 0.57 to $-0.57 \mathrm{~V}$ and then back to $0.57 \mathrm{~V}$ during imaging ( $I$ $=0.6 \mathrm{nA}$ and image size: $4.4 \mathrm{~nm} \times 4.4 \mathrm{~nm})$. The tip-field $(E)$-induced mono-

mer reorientations are schematically illustrated. When scanning along the chain axis. This anisotropy in the
Downloaded 05 Sep 2006 to 129.93 .16 .206 . Redistribution subject to AIP license or copyright, see http://apl.aip.org/apl/copyright.jsp

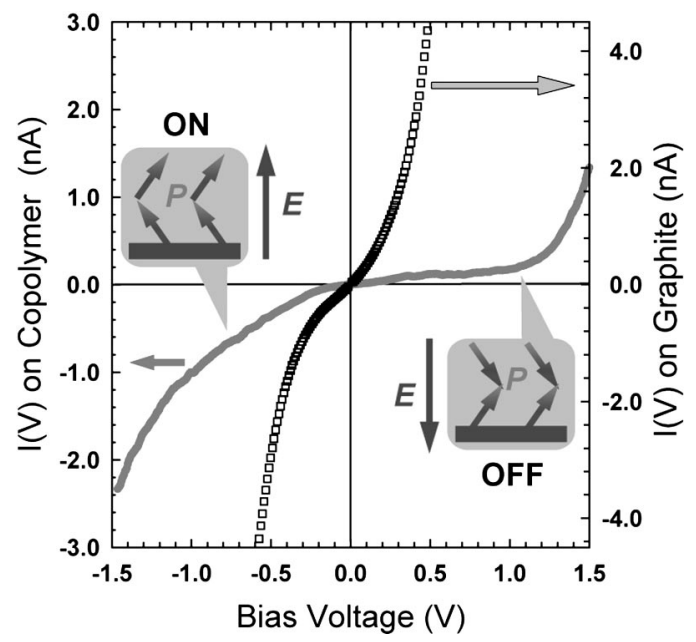

FIG. 3. An averaged tunneling current $I(V)$ as a function of tip bias voltage taken at the top of 50 monomer protrusions of a 2-ML $P$ (VDF-TrFE) film on graphite at RT, compared with that taken from the graphite substrate alone with exactly the same measurement conditions. The "ON/OFF" state occurs when the top-layer dipoles $(P)$ that are rotated by the tip field $(E)$ follow/oppose the bottom-layer dipoles that are pinned by the substrate (see insets with the arrows representing dipole moments).

will force the local dipoles underneath the tip to orient along the surface normal with the charge-positive hydrogen towards the vacuum, while a positive bias induces the dipoles to polarize into the opposite direction, resulting in necessary local dipole rotation or lattice distortion.

One possible mechanism for the rotation is that flipping the STM bias completely flips the dipole moment positioned right underneath the tip, but twists nearby monomer moments partially with some canting angle $\theta$. In the plane perpendicular to the chains, a rotation by $\pi / 2$ in one $-\mathrm{C}-\mathrm{C}-$, followed another $\pi / 2$ in next $-\mathrm{C}-\mathrm{C}$-bond, would result in a dipole reversal that remains in the surface plane. Such a dipole reversal requires a minimal chain length, but does result in a displacement. The displacement in chain position due to the induced canting then appears in the STM images. In this case, surface dipole moments are largely normal to the surface prior to the flipping, as suggested from the symmetry arguments based upon the results of photoelectron and other spectroscopy measurements. ${ }^{18,19}$

The other scenario is that the tip field locally induces a local polarization reversal of the surface layer from one dipole-canted phase to another but does not completely flip the dipole moments. In the negative tip-bias image (corresponding to the middle part of the image of Fig. 2), the surface dipoles are canted with an angle $\theta$ relative to the surface normal and the imaged protrusions represent the $-\mathrm{CH}_{2} / \mathrm{CHF}-$ subclusters. When tip bias is flipped to positive the reversed field forces the local dipoles to rotate by amount of $\pi-2 \theta$ about the chain axis. These dipoles are canted inward with an angle of $\pi-\theta$ relative to the surface normal and the imaged protrusions represent the $-\mathrm{CF}_{2}-$ subclusters. The rotation directly results in a lateral lattice shift perpendicular to the chain axis in the spontaneous image (Fig. 2).

Since each dipole is still normal to chain axis during the rotation in either scenario, no intrachain lattice shift by flipping should be expected, consistent with what we observed when scanning along the chain axis. This anisotropy in the 
imaged lattice shift clearly indicates that the dipole moments rotate only in the plane perpendicular to the chain axis; that is, with restricted freedom. When the tip bias is below a certain value $(\sim 0.1 \mathrm{~V})$, the shift will not occur since the tip field is below the critical polarization field for rotation; that is, the interaction energy between a dipole and external field is not enough to overcome the energy barrier for the rotation. On the other hand, when the tip bias is over a certain value, the large tip field will completely polarize the top layer (at least locally) without canting, thus, any apparent shift in imaged lattices by flipping bias should not be expected. This explains why the shift disappeared when $\left|V_{\text {bias }}\right|$ exceeded a certain value $(\sim 1.0 \mathrm{~V})$. Given a low limit of $V_{\text {bias }} \sim 0.1 \mathrm{~V}$ for the induced dipole rotation, a critical electric field of $E_{\text {cr }}$ $\sim 0.2-0.4 \mathrm{GV} \mathrm{m}^{-1}$ is estimated (when the tip-sample gap is $\sim 3-5 \AA$ ), comparable with the coercive field of $\sim 0.5$ $\mathrm{GV} \mathrm{m}^{-1}$ for a 2-ML film obtained by the pyroelectric technique. ${ }^{11}$

To directly characterize the response of transport properties associated with the local induced polarization reversal, we have performed scanning tunneling spectroscopy on individual monomer protrusions. We found that the tunneling current $I(V)$ as a function of $V_{\text {bias }}$ was stable and quite repeatable on different monomer protrusions. We also observed a negligible difference in $I(V)$ when the tip was displaced from the central location of protrusions. However, a local "switching" phenomenon in vertical conductance was clearly observed with flipping the polarity of $V_{\text {bias }}$. As shown in Fig. 3, the $I(V)$ are quite asymmetric in the bias range of $\left|V_{\text {bias }}\right| \leqslant 1.2 \mathrm{~V}$, behaving as a diode character. A much larger tunneling current was observed with negative $V_{\text {bias }}$ as compared with that with positive one. The change of the conductance with polarization reversal in the bias range of $0.2 \mathrm{~V}$ $\leqslant\left|V_{\text {bias }}\right| \leqslant 1.0 \mathrm{~V}$ is more than six times, and is similar to that observed with adsorbed boron subphalocyanine molecules. ${ }^{7}$

There are two possible contributions to such asymmetric behavior. One is simply the effect on $I(V)$ due to the different density of states between the filled and empty states and, because the Fermi level is close to the conduction band, the film behaves as an $n$-type semiconductor. ${ }^{20}$ The other possibility is the change of interfacial potential barrier caused by the induced local polarization reversal of the top layer. It is worthwhile to mention that the bias voltage range $\left(\mid V_{\text {bias }}\right.$ $\mid \leqslant 1.2 \mathrm{~V}$ ) for the asymmetric behavior in the $I(V)$ (see Fig. 3 ) is also the range for the dipole reversal shown in Fig. 2 (except the low bias $\left|V_{\text {bias }}\right| \leqslant 0.1 \mathrm{~V}$ ). Furthermore, this voltage range is very close to that for the macroscopic polarization reversal measurements for the similar thin films. ${ }^{10}$

It is likely that the interfacial potential barrier is regulated by the relative orientations of dipole moments between the top and bottom layer. The alignment between these two layer dipoles offers either "ON" or "OFF" switching state (Fig. 3). In these $P(\mathrm{VDF}-\mathrm{TrFE})$ crystalline thin films, the dipole moments in the bottom layer are pinned directly by the substrate while the dipole moments in the top "free" layer can follow the external polarization field. The pinning effect by the substrate has been indicated with the exchangebias behavior in the polarization hysteresis loop measured from the same thickness of films. ${ }^{10,11}$ This may be due to the stronger interaction between the bottom layer of polymer and the substrate when the electronegative fluorine atoms rather than electropositive hydrogen atoms are attached to the substrate surface, which can play a role as a charge donor. When the polarization in the top layer manipulated by the external tip field is against that in the bottom pinned layer, in contrast with the ferroelectric alignment between top and bottom layers, an interfacial potential barrier will be built up to reduce the vertical conductance. Only when a large-enough external field is applied and eventually reverses the polarization of the pinned layer (at least locally), a "reversal breakdown" will occur similar to a $p-n$ junction diode. The breakdown voltage of $\sim 1.2 \mathrm{~V}$ estimated from the $I(V)$ spectrum (Fig. 3) corresponds to an exchange bias of $\sim 1.2 \mathrm{GV} \mathrm{m}^{-1}$. This is comparable with the earlier measured value of $\sim 1.0$ $\mathrm{GV} \mathrm{m}^{-1}$. $^{11}$

The support of the Petroleum Research Fund, administered by the ACS, the Office of Naval Research, and the Nebraska Research Initiative are gratefully acknowledged.

${ }^{1}$ M. A. Ratner, Materialstoday 5, 20 (2002), and the references therein.

${ }^{2}$ A. Aviram, C. Joachim, and M. Pomerantz, J. Am. Chem. Soc. 110, 5687 (1988).

${ }^{3}$ R. S. Potember, T. O. Poehler, and D. O. Cowan, Appl. Phys. Lett. 34, 405 (1979); R. S. Poetmber, T. O. Poehler, and R. C. Benson, Appl. Phys. Lett. 41, 548 (1982).

${ }^{4}$ H. J. Gao, K. Sohlberg, Z. Q. Xue, H. Y. Chen, S. M. Hou, L. P. Fang, S. J. Pang, and S. J. Pennycook, Phys. Rev. Lett. 84, 1780 (2000).

${ }^{5}$ H. Yanagi, K. Ikuta, H. Mukai, and T. Shibutani, Nano Lett. 2, 951 (2002).

${ }^{6}$ Y. Zhao, A. Fein, C. A. Peterson, and D. Sarid, Phys. Rev. Lett. 87, 179706 (2001).

${ }^{7}$ T. Furukawa, Phase Transitions 18, 143 (1989).

${ }^{8}$ S. Palto, L. Blinov, A. Bune, E. Dubovik, V. M. Fridkin, N. Petuknova, K. Verkhovskaya, and S. Yudin, Ferroelectr. Lett. 19, 65 (1995).

${ }^{9}$ S. Palto, L. Blinov, A. Bune, E. Dubovik, V. M. Fridkin, N. Petuknova, A. Sorokin, K. Verkhovskaya, S. Yudin, and A. Zlatkin, Europhys. Lett. 34, 465 (1996).

${ }^{10}$ A. V. Bune, V. M. Fridkin, S. Ducharme, L. M. Blinov, S. P. Palto, A. V. Sorokin, S. Yudin, and A. Zlatkin, Nature (London) 391, 874 (1998).

${ }^{11}$ L. M. Blinov, V. M. Fridkin, S. P. Palto, A. V. Bune, P. A. Dowben, and S. Ducharme, Phys. Usp. 43, 243 (2000), and the references therein.

${ }^{12}$ A. V. Bune, S. Ducharme, V. M. Fridkin, L. M. Blinov, S. P. Palto, N. Petuknova, and S. Yudin, Appl. Phys. Lett. 67, 3975 (1995).

${ }^{13}$ S. Ducharme, V. M. Fridkin, A. V. Bune, S. P. Palto, L. M. Blinov, N. N. Petukhova, and S. G. Yudin, Phys. Rev. Lett. 84, 175 (2000).

${ }^{14}$ D. R. Tilley, in Ferroelectric Thin Films: Synthesis and Basic Properties, edited by C. Paz de Araujo, J. F. Scott, and G. F. Taylor (Gordon and Breach, Amsterdam, 1996), pp. 11-45.

${ }^{15}$ B. D. Qu, P. L. Zhang, Y. G. Wang, C. L. Wang, and W. L. Zhong, Ferroelectrics 152, 219 (1994).

${ }^{16}$ J. Choi, C. N. Borca, P. A. Dowben, A. Bune, M. Poulsen, S. Pebley, S. Adenwalla, S. Ducharme, L. Robertson, V. M. Fridkin, S. P. Palto, N. N. Petukhova, and S. G. Yudin, Phys. Rev. B 61, 5760 (2000).

${ }^{17}$ E. Bellet-Amalric and J. F. Legrand, Eur. Phys. J. B 3, 225 (1998).

${ }^{18}$ Jaewu Choi, S.-J. Tang, P. T. Sprunger, P. A. Dowben, V. M. Fridkin, A. V. Sorokin, S. P. Palto, N. Petukhova, and S. G. Yudin, J. Phys.: Condens. Matter 12, 4735 (2000).

${ }^{19}$ Mengjun Bai, Ph.D. thesis, University of Nebraska, 2002.

${ }^{20}$ J. Choi, P. A. Dowben, S. Pebley, A. V. Bune, and S. Ducharme, Phys. Rev. Lett. 80, 1328 (1998). 